\title{
FIELDS IN TERMS OF A SINGLE OPERATION
}

\author{
BY \\ ROBERT J. LEVIT
}

1. Introduction. The customary conception of a field is a system based on two inherently independent operations and thereby sharply differentiated from systems of single composition such as groups. That this distinction is not essential was first demonstrated by $\mathrm{N}$. Wiener $\left({ }^{1}\right)$ when he exhibited a postulate set for fields requiring only one primitive operation $\left({ }^{2}\right)$. This operation, here designated as $a \nabla b$, is expressible in terms of the usual field operations as $1-a / b . \nabla$ possesses the remarkable property that every rational binary operation of the field as well as the zero and unit elements can be directly expressed in terms of it. However, it has the disadvantage that the field is not closed under it. The absence of closure is always a source of much complexity in the use of an operation because it is necessary to determine for each expression the conditions under which it belongs to the class and to treat separately the exceptional cases that arise when it does not belong.

In the present paper a definition of field is given by means of five independent postulates in terms of a single operation which is class-closing. This definition will be referred to as (F) and its primitive operation as $\Delta . a \Delta b$ may be expressed as $a \cdot(1-b)$. The two operations $\Delta$ and $\nabla$ are intimately related, since $\nabla$ is the right inverse of $\Delta$. A second definition $\left(F^{\prime}\right)$ of field in terms of $\nabla$ is given by means of six independent postulates. It is used to give a brief demonstration of the sufficiency of $(F)$. The sufficiency of $\left(F^{\prime}\right)$ is established by recourse to Wiener's postulates. In $\$ \S 2-8$ the postulates are stated and their sufficiency, necessity, and independence proved. Certain additional properties of the operations are stated in $\S 9$. In $\$ 10$ it is shown that $(\Delta)$ and $(\nabla)$ are susceptible of a variety of interpretations in terms of the field sum and product, and all such interpretations are found. In $\$ 11$ are listed a number of other single operations that can be used to define field.

At this time I should like to express my appreciation to Professor B. A. Bernstein for the interest he showed and the many valuable suggestions he gave me in the preparation of this paper.

2. Postulate set (F). Consider as primitive a class $K$ and a binary operation $(\Delta)$. The postulates $\mathrm{F}_{1}-\mathrm{F}_{5}$ following will make the system $(K, \Delta)$ a field.

Presented to the Society, November 22, 1941; received by the editors January 14, 1945.

(1) Norbert Wiener, $A$ set of postulates for fields, Trans. Amer. Math. Soc. vol. 21 (1920) pp. 237-246.

(2) On the other hand a group can be regarded as a system of double composition. See H. Boggs and G. Y. Rainich, Note on group postulates, Bull. Amer. Math. Soc. vol. 43 (1937) pp. 81-84. 
Definitions are denoted by ' $D$ '. In $F_{2}, F_{3}$, and $F_{5}$, the condition, "provided the elements involved and their indicated combinations are in $K$," is to be understood.

$F_{1}$. For every $a$ and $b$ in $K, a \Delta b$ is an element of $K$.

$\mathrm{F}_{2} .(a \Delta b) \Delta c=(a \Delta c) \Delta b$.

$\mathrm{F}_{3} . a \Delta b=a$ implies that $b \Delta a=b$.

$\mathrm{D}_{1}$. If 0 is a $K$-element such that $a \Delta 0=a$ for every $a$ in $K, 0$ is called a zero-element.

$\mathrm{F}_{4}$. If $a$ and $b$ are $K$-elements, there is an element $x$ in $K$ such that $a \Delta x=b$, unless $K$ contains a unique zero-element 0 and $a=0$.

$\mathrm{D}_{2}$. If $K$ contains a unique zero-element 0 , and 1 is a $K$-element such that $a \Delta 1=0$ for every $a$ in $K, 1$ is called a unity-element.

$F_{5}$. If 1 is any unity-element, $1 \Delta[a \Delta(b \Delta c)]=b$ implies that $1 \Delta[b \Delta(a \Delta c)]$ $=a$.

There are no categorical existence postulates in the above set. If it is desired to exclude the trivial empty and one-element classes from consideration as fields, the following must be added.

N. $K$ contains at least two elements.

3. Elementary theorems from (F). The following theorems will be used to established the sufficiency of $(\mathrm{F})$ for fields. Postulate $\mathrm{N}$ will be assumed and used in the proofs without citation.

$\mathrm{T}_{1} . K$ contains at least one zero-element.

Proof. Let $a$ and $b$ be any $K$-elements. We may suppose that $a$ is not a zero-element, since otherwise there would be nothing to prove. By $\mathrm{F}_{4}$ there exist elements $z$ and $x$ such that

$$
a \Delta z=a,
$$$$
b=a \Delta x .
$$

Then, $b \Delta z=(a \Delta x) \Delta z=(a \Delta z) \Delta x=a \Delta x=b$; by (2), $\mathrm{F}_{2},(1),(2)$. Hence, since $b$ was arbitrary, $z$ is a zero-element by $D_{1}$.

$\mathrm{T}_{2} .0 \Delta a=0$, where 0 is any zero-element.

Proof. $a \Delta 0=a$ for every $a$ by $\mathrm{D}_{1}$. Hence, $0 \Delta a=0$; by $\mathrm{F}_{3}$.

$\mathrm{T}_{3}$. There is a unique zero-element 0 in $K$.

Proof. There is at least one zero-element by $T_{1}$. Suppose there were more than one, and let 0 and $z$ be distinct zero-elements. Then by $\mathrm{F}_{4}$, for every $a$ and $b$ there would exist an $x$ such that $a \Delta x=b$. In particular, there would be an $x$ such that $z \Delta x=0$. But this is impossible, since $z \Delta x=z \neq 0$ by $T_{2}$. Hence 0 is unique.

$\mathrm{T}_{4} . K$ contains at least one unity-element.

Proof. Let $a \neq 0, b$ any $K$-element. By $\mathrm{F}_{4}$ there are elements $u, x$ such that 


$$
a \Delta u=0,
$$

$$
b=a \Delta x .
$$

Then, $b \Delta u=(a \Delta x) \Delta u=(a \Delta u) \Delta x=0 \Delta x=0$; by (2), $\mathrm{F}_{2},(1), \mathrm{T}_{2}$. Hence, since $b$ was arbitrary, $u$ is a unity-element by $D_{\mathbf{2}}$.

$T_{5} .1 \Delta(a \Delta b)=b$ implies that $1 \Delta(b \Delta a)=a$, where 1 is any unity-element.

Proof. $b=1 \Delta(a \Delta b)=1 \Delta[a \Delta(b \Delta 0)]$; by hypothesis, $D_{1}$. Therefore $a$ $=1 \Delta[b \Delta(a \Delta 0)]=1 \Delta(b \Delta a) ;$ by $\mathrm{F}_{5}, \mathrm{D}_{1}$.

$\mathrm{T}_{6} .1 \Delta(1 \Delta a)=a$, where 1 is any unity-element.

Proof. $1 \Delta(a \Delta 1)=1 \Delta 0=1$; by $\mathrm{D}_{2}, \mathrm{D}_{1}$. Hence, $1 \Delta(1 \Delta a)=a$; by $\mathrm{T}_{6}$.

$\mathrm{T}_{7}$. There is a unique unity-element 1 in $K$.

Proof. There is at least one unity-element by $T_{4}$. Let 1 and $u$ be unityelements. Then, $u=1 \Delta(1 \Delta u)=1 \Delta 0=1$; by $\mathrm{T}_{6}, \mathrm{D}_{2}, \mathrm{D}_{1}$.

T8. $1 \neq 0$.

Proof. Suppose $1=0$ and let $a \neq 0$. Then $a=a \Delta 0=a \Delta 1=0$; by $\mathrm{D}_{1}$, hypothesis, $\mathrm{D}_{2}$-contrary to the assumption that $a \neq 0$.

$\mathrm{T}_{9}$. If $a \Delta x=a \Delta y$ and $a \neq 0$, then $x=y$.

Proof. Let $a \neq 0$ and $a \Delta x=a \Delta y$ (1). By $\mathrm{F}_{4}$ there is an element $\bar{a}$ such that $a \Delta \bar{a}=1$ (2). Then, $x=1 \Delta(1 \Delta x)=1 \Delta[(a \Delta \bar{a}) \Delta x]=1 \Delta[(a \Delta x) \Delta \bar{a}]=1 \Delta[(a \Delta y) \Delta \bar{a}]$ $=1 \Delta[(a \Delta \bar{a}) \Delta y]=1 \Delta(1 \Delta y)=y$; by $\mathrm{T}_{6},(2), \mathrm{F}_{2},(1), \mathrm{F}_{2},(2), \mathrm{T}_{6}$.

$\mathrm{T}_{10}$. The equation $a \Delta x=b$ has a unique solution $x$ if and only if $a \neq 0$.

Proof. If $a \neq 0$, there is a unique solution by $\mathrm{F}_{4}, \mathrm{~T}_{9}$. Conversely, $0 \Delta x=0$ for every $x$; by $\mathrm{T}_{2}$. Hence, if $a=0$, the equation has no solution unless $b=0$ also, and in that case it is satisfied by every $K$-element.

$\mathrm{D}_{3} . a \nabla b$ denotes the element $x$ such that $b \Delta x=a$, whenever $x$ is uniquely determined by $a$ and $b$.

$T_{11} . a \nabla b$ is an element of $K$ if and only if $b \neq 0$.

Proof. The proof is by $\mathrm{D}_{3}, \mathrm{~T}_{10}$.

$\mathrm{T}_{12 .} . a \nabla a=0,0 \nabla a=1$, where $a \neq 0$.

Proof. Let $p=a \nabla a$. Then, $a \Delta p=a=a \Delta 0$; by $\mathrm{D}_{3}, \mathrm{~T}_{3}$. Hence, since $a \neq 0$ by hypothesis, $p=0$; by $\mathrm{T}_{9}$. Next, let $q=0 \nabla a$. Then $a \Delta q=0=a \Delta 1$; by $\mathrm{D}_{3}, \mathrm{~T}_{7}$. Hence, since $a \neq 0$ by hypothesis, $q=1$, by $\mathrm{T}_{9}$.

$T_{13}$. If $a$ and $b$ are distinct $K$-elements and $b \neq a \nabla a$, then $a \nabla b$ is an element of $K$.

Proof. If $a \neq 0, a \nabla a=0$ by $\mathrm{T}_{12}$. Hence $b \neq 0$ by hypothesis. If $a=0, b \neq 0$, 
since $a$ and $b$ are distinct by hypothesis. In either case, $b \neq 0$. Hence $a \nabla b$ is in $K$ by $T_{11}$.

$T_{14}$. If $b \nabla b$ is not an element of $K$, neither is $a \nabla b$.

Proof. If $b \nabla b$ is not in $K, b=0$; by $\mathrm{T}_{11}$. Hence $a \nabla b$ is not in $K$; by $\mathrm{T}_{11}$.

$\mathrm{T}_{15}$. If $a \nabla b=c \nabla d$ and $b, c, d \neq 0$, then $a \nabla c=b \nabla d$.

Proof. Let $p=a \nabla b=c \nabla d(1) ; q=a \nabla c(2) ; r=b \nabla d$ (3). Then by $\mathrm{D}_{3}, a=b \Delta p$ (4); $c=d \Delta p$ (5); $a=c \Delta q$ (6); $b=d \Delta r$ (7). Therefore $c \Delta q=a=b \Delta p=(d \Delta r) \Delta p$ $=(d \Delta p) \Delta r=c \Delta r$; by (6), (4), (7), $\mathrm{F}_{2},(5)$. Hence, since $c \neq 0$ by hypothesis, $q=r$; by $\mathrm{T}_{9}$; that is, $a \nabla c=b \nabla d$; by (2), (3).

$\mathrm{T}_{16 .}(a \nabla a) \nabla b=(b \nabla b) \nabla a$, where $a, b \neq 0$.

Proof. $(a \nabla a) \nabla b=0 \nabla b=1=0 \nabla a=(b \nabla b) \nabla a$; by $\mathrm{T}_{12}, \mathrm{~T}_{12}, \mathrm{~T}_{12}, \mathrm{~T}_{12}$.

$\mathrm{T}_{17}$. If $x \nabla a=y \nabla a, x=y$, where $a \neq 0$.

Proof. Let $x \nabla a=p=y \nabla a$. Then $x=a \Delta p=y$; by $\mathrm{D}_{3}$.

$\mathrm{D}_{4} . a^{\prime}=1 \Delta a$.

$\mathrm{T}_{18 .}\left(a^{\prime} \nabla b\right) \nabla a=\left(b^{\prime} \nabla a\right) \nabla b$, where $a, b \neq 0$.

Proof. Let $p=a^{\prime} \nabla b(1) ; q=p \nabla a(2) ; r=b^{\prime} \nabla a(3) ; s=r \nabla b$ (4). Then, $a^{\prime}=b \Delta p$ (5); $p=a \Delta q$ (6); $b^{\prime}=a \Delta r$ (7); $r=b \Delta s$ (8); by $\mathrm{D}_{3}$ and $1 \Delta a=b \Delta(a \Delta q)$ (9); $1 \Delta b=a \Delta(b \Delta s) \quad(10)$; by $\mathrm{D}_{4}, \quad(5)-(8) . a=1 \Delta(1 \Delta a)=1 \Delta[b \Delta(a \Delta q)]$ (11); and $b=1 \Delta(1 \Delta b)=1 \Delta[a \Delta(b \Delta s)](12) ;$ by $\mathrm{T}_{6},(9),(10)$. Therefore $1 \Delta[a \Delta(b \Delta q)]=b$ $=1 \Delta[a \Delta(b \Delta s)]$; by (11), $\mathrm{F}_{5},(12)$. Hence $q=s$; by ' $\mathrm{T}_{8}, \mathrm{~T}_{9}, \mathrm{~T}_{9}, \mathrm{~T}_{9}$ and $\left(a^{\prime} \nabla b\right) \nabla a$ $=q=s=\left(b^{\prime} \nabla a\right) \nabla b$; by (1)-(4).

4. Postulate set $\left(F^{\prime}\right)$. The last six theorems in $\S 3$ can be taken as the basis of a convenient definition of fields in terms of the operation $(\nabla)$, the inverse of $(\Delta)$. Consider as primitive a class $K$ and a binary operation $\nabla$. The postulates $F_{1}^{\prime}-\mathrm{F}_{6}^{\prime}$ following will make the system $(K, \nabla)$ a field. In $\mathrm{F}_{3}^{\prime}, \mathrm{F}_{4}^{\prime}, \mathrm{F}_{5}^{\prime}, \mathrm{F}_{6}^{\prime}$ and $\mathrm{D}_{1}^{\prime}$ the condition, "provided the elements involved and their indicated combinations are in $K$," is to be understood. To exclude trivial cases, postulate $\mathrm{N}$ must again be added. See $\$ 2$.

$\mathrm{F}_{1}^{\prime}$. If $a$ and $b$ are distinct $K$-elements and $b \neq a \nabla a$, then $a \nabla b$ is an element of $K$.

$\mathrm{F}_{2}^{\prime}$. If $a$ and $b$ are $K$-elements and $b \nabla b$ is not an element of $K$, then $a \nabla b$ is not an element of $K$.

$\mathrm{F}_{3}^{\prime} . a \nabla b=c \nabla d$ implies that $a \nabla c=b \nabla d$.

$\mathrm{F}_{4}^{\prime} .(a \nabla a) \nabla b=(b \nabla b) \nabla a$.

$\mathrm{F}_{\mathrm{b}}^{\prime} . x \nabla a=y \nabla a$ implies that $x=y$.

$\mathrm{D}_{1}^{\prime} \cdot a^{\prime}=a \nabla[(a \nabla a) \nabla a]$.

$\mathrm{F}_{6}^{\prime} .\left(a^{\prime} \nabla b\right) \nabla a=\left(b^{\prime} \nabla a\right) \nabla b$. 
5. Elementary theorems from $\left(\mathbf{F}^{\prime}\right)$. The following will be used to establish the sufficiency of $\left(\mathrm{F}^{\prime}\right)$ for fields. Postulate $\mathrm{N}$ will be assumed without citation. Certain obvious steps in the proofs have been left to the reader.

$T_{1}^{\prime}$. There is an element of the form $a \nabla a$ in $K$.

Proof. Let $a$ and $b$ be distinct $K$-elements and suppose $b \nabla b$ not in $K$. Then $a \nabla b$ is not in $K$; by $\mathrm{F}_{2}^{\prime}$. Hence, by $\mathrm{F}_{1}^{\prime}, a \nabla a=b$ and so is an element of $K$.

$\mathrm{T}_{2}^{\prime}$. If $a$ and $b$ are distinct and $b=a \nabla a$, then $a \neq b \nabla b$.

Proof. Let $a \nabla a=b$ (1), $b \neq a$ (2), and suppose that $b \nabla b=a$ (3). Then, $b=a \nabla a=(b \nabla b) \nabla a=(a \nabla a) \nabla b=b \nabla b=a$; by (1), (3), $\mathrm{F}_{4}^{\prime}$, (1), (3) contradicting (2). Hence (3) is impossible.

$T_{3}^{\prime} . a \nabla a=b \nabla b$ (whenever $a \nabla a$ and $b \nabla b$ are elements of $K$ ).

Proof. Let $a \neq b$; then, by $\mathrm{T}_{2}^{\prime}, b=a \nabla a$ and $a=b \nabla b$ cannot both hold. Suppose $b \neq a \nabla a$. Then $a \nabla b$ is in $K$; by $\mathrm{F}_{1}^{\prime}$, and, from $a \nabla b=a \nabla b$ follows $a \nabla a=b \nabla b$; by $F_{3}^{\prime}$.

$\mathrm{D}_{2}^{\prime} .0=a \nabla a$, where $a$ is any $K$-element such that $a \nabla a$ is in $K$. (0 is a uniquely defined $K$ element by $\mathrm{T}_{1}^{\prime}, \mathrm{T}_{3}^{\prime}$.)

$\mathrm{T}_{4}^{\prime} . a \nabla b$ is an element of $K$ if and only if $b \neq 0$.

Proof. Let $a$ and $b$ be distinct $K$-elements and $b \neq 0$. Then $b \neq a \nabla a$; by $\mathrm{D}_{2}^{\prime}$. Hence $a \nabla b$ is an element of $K$; by $\mathrm{F}_{1}^{\prime} . b \nabla b$ is also an element of $K$; by $\mathrm{F}_{2}^{\prime}$. Conversely, suppose $a \nabla 0$ were in $K$. Then $0 \nabla 0$ would be in $K$; by $\mathrm{F}_{2}^{\prime}$. Let $b \neq 0(1)$. Then $b \nabla b$ and $0 \nabla b$ are in $K$; by the first part of this theorem. Therefore $b \nabla b=0 \nabla 0=(b \nabla b) \nabla 0=(0 \nabla 0) \nabla b=0 \nabla b$; by $\mathrm{T}_{3}^{\prime}, \mathrm{D}_{2}^{\prime}, \mathrm{F}_{4}^{\prime}, \mathrm{D}_{2}^{\prime}$. Therefore $b=0$; by $F_{b}^{\prime}$, contradicting (1). Hence $0 \nabla 0$ and consequently $a \nabla 0$ are not elements of $K$.

$\mathrm{T}_{6}^{\prime} .0 \nabla a=0 \nabla b$ (where $\left.a, b \neq 0\right)$.

Proof. $a \nabla a, b \nabla b,(a \nabla a) \nabla b$, and $(b \nabla b) \nabla a$ are in $K$; by $\mathrm{T}_{4}^{\prime}$. Therefore $0 \nabla a$ $=(b \nabla b) \nabla a=(a \nabla a) \nabla b=0 \nabla b ;$ by $\mathrm{D}_{2}^{\prime}, \mathrm{F}_{4}^{\prime}, \mathrm{D}_{2}^{\prime}$.

$\mathrm{D}_{3}^{\prime} .1=0 \nabla a$, where $a \neq 0$. ( 1 is a uniquely defined $K$-element; by $\mathrm{T}_{4}^{\prime}, \mathrm{T}_{5}^{\prime}$.)

$\mathrm{T}_{6}^{\prime}$. If $a \nabla b=0, a=b$ (where $\left.b \neq 0\right)$.

Proof. Let $b \neq 0$ and $a \nabla b=0$ (1). Then $b \nabla b$ is in $K$ by $T_{4}^{\prime} ;$ and $a \nabla b=0=b \nabla b$; by (1), $\mathrm{D}_{2}^{\prime}$. Hence, $a=b$; by $\mathrm{F}_{5}^{\prime}$.

$\mathrm{T}_{7}^{\prime} .1 \neq 0$.

Proof. Suppose $1=0$ (1), and let $a \neq 0$ (2). Then $a \nabla a$ is in $K_{;}$by $\mathrm{T}_{4}^{\prime}$. $a \nabla a=0=1=0 \nabla a$; by $\mathrm{D}_{2}^{\prime},(1), \mathrm{D}_{3}^{\prime}$. Hence $a=0$ by $\mathrm{F}_{b}^{\prime}$, contradicting (2).

$\mathrm{T}_{8}^{\prime}$. If $a \nabla b=c \nabla d, b \nabla a=d \nabla c$ (where $\left.a, b, c, d \neq 0\right)$. 
Proof. From $a \nabla b=c \nabla d$ follows $a \nabla c=b \nabla d$ by $\mathrm{F}_{3}^{\prime}$. Writing this as $b \nabla d=a \nabla c$, it follows that $b \nabla a=d \nabla c$ by $\mathrm{F}_{3}^{\prime}$.

$\mathrm{D}_{4}^{\prime} \cdot 0^{\prime}=1$. (In view of $\mathrm{T}_{4}^{\prime}, 0^{\prime}$ is not defined by $\mathrm{D}_{1}^{\prime}$.)

$\mathrm{T}_{0}^{\prime} . a^{\prime}=a \nabla 1$.

Proof. For $a=0,0^{\prime}=1=0 \nabla 1$ by $\mathrm{D}_{4}^{\prime}, \mathrm{D}_{3}^{\prime}$. For $a \neq 0, a^{\prime}=a \nabla[(a \nabla a) \nabla a]$ $=a \nabla(0 \nabla a)=a \nabla 1 ;$ by $\mathrm{D}_{1}^{\prime}, \mathrm{D}_{2}^{\prime}, \mathrm{D}_{3}^{\prime}$.

$\mathrm{T}_{10}{ }^{\prime} \cdot 1^{\prime}=0$.

Proof. $1^{\prime}=1 \nabla 1=0$; by $\mathrm{T}_{9}^{\prime}, \mathrm{D}_{2}^{\prime}$.

$\mathrm{D}_{5}^{\prime} . a^{\prime \prime}=\left(a^{\prime}\right)^{\prime}$.

Note. References tc $\mathrm{T}_{1}^{\prime}-\mathrm{T}_{10}^{\prime}$ and $\mathrm{D}_{1}^{\prime}-\mathrm{D}_{6}^{\prime}$ will frequently be omitted when obvious.

$\mathrm{T}_{11}^{\prime} \cdot a^{\prime \prime}=a$.

Proof. For $a=0$, the theorem is obvious by $\mathrm{D}_{4}^{\prime}, \mathrm{T}_{10}^{\prime}$. Let $a \neq 0 . a^{\prime \prime} \nabla a$ $=\left(a^{\prime} \nabla 1\right) \nabla a=\left(1^{\prime} \nabla a\right) \nabla 1=(0 \nabla a) \nabla 1=1 \nabla 1=0$ by $\mathrm{T}_{9}^{\prime}, \mathrm{F}_{6}^{\prime}, \mathrm{T}_{10}^{\prime}, \mathrm{D}_{3}^{\prime}, \mathrm{D}_{2}^{\prime}$. Hence $a^{\prime \prime}=a$ by $\mathrm{T}_{6}^{\prime}$.

$\mathrm{T}_{12}^{\prime}$. If $(a \nabla b)^{\prime}=0, a=0$ (where $\left.b \neq 0\right)$.

Proof. $0=(a \nabla b)^{\prime}=(a \nabla b) \nabla 1$. Hence $a \nabla b=1=0 \nabla b$ and $a=0$ by $\mathrm{T}_{6}^{\prime}, \mathrm{F}_{6}^{\prime}$.

$\mathrm{T}_{13}^{\prime} \cdot a \nabla(a \nabla b)^{\prime}=b^{\prime}$ (where $\left.a, b \neq 0\right)$.

Proof. Since $a \neq 0,(a \nabla b)^{\prime} \neq 0$; by $\mathrm{T}_{12}^{\prime}$. Then $a \nabla b=(a \nabla b)^{\prime \prime}=(a \nabla b)^{\prime} \nabla 1$ by $\mathrm{T}_{11}^{\prime}, \mathrm{T}_{9}^{\prime}$. Hence $a \nabla(a \nabla b)^{\prime}=b \nabla 1=b^{\prime}$ by $\mathrm{F}_{3}^{\prime}, \mathrm{T}_{9}^{\prime}$.

$\mathrm{T}_{14}^{\prime} \cdot(a \nabla c)^{\prime} \nabla(b \nabla c)^{\prime}=a \nabla b$ (where $\left.b, c \neq 0\right)$.

Proof. For $a=0$ the theorem is obvious. Let $a, b, c \neq 0$. Then $(a \nabla c)^{\prime} \neq 0$, $(b \nabla c)^{\prime} \neq 0$ by $\mathrm{T}_{12}^{\prime} \cdot a \nabla(a \nabla c)^{\prime}=c^{\prime}=b \nabla(b \nabla c)^{\prime}$ by $\mathrm{T}_{13}^{\prime}, \mathrm{T}_{13}^{\prime} . a \nabla b=(a \nabla c)^{\prime} \nabla(b \nabla c)^{\prime}$ by $\mathrm{F}_{3}^{\prime}$.

$\mathrm{T}_{16^{\prime}} \cdot(a \nabla b)^{\prime} \nabla c=(a \nabla c)^{\prime} \nabla b$ (where $b, c \neq 0$ ).

Proof. For $a=0$, the theorem is obvious. Let $a \neq 0$. Since $a, c \neq 0,(a \nabla b)^{\prime} \neq 0$, $(a \nabla c)^{\prime} \neq 0,(c \nabla b)^{\prime} \neq 0$ by $\mathrm{T}_{12}^{\prime}$. Then, $(a \nabla b)^{\prime} \nabla(a \nabla c)^{\prime}=(a \nabla b)^{\prime} \nabla\left[(a \nabla b)^{\prime} \nabla(c \nabla b)^{\prime}\right]^{\prime}$ $=\left[(c \nabla b)^{\prime}\right]^{\prime}=c \nabla b$ by $\mathrm{T}_{14}^{\prime}, \mathrm{T}_{13}^{\prime}, \mathrm{T}_{11}^{\prime}$. Therefore $(a \nabla b)^{\prime} \nabla c=(a \nabla c)^{\prime} \nabla b$ by $\mathrm{F}_{3}^{\prime}$.

$\mathrm{T}_{16^{\prime}} \cdot[(a \nabla b) \nabla(0 \nabla b)] \nabla c=[(a \nabla c) \nabla(0 \nabla c)] \nabla b$ where $b, c \neq 0$.

Proof. This is proved by $\mathrm{D}_{3}^{\prime}, \mathrm{T}_{9}^{\prime}, \mathrm{T}_{16}^{\prime}$.

$\mathrm{D}_{6}^{\prime} \cdot a \cdot b=\left[a \nabla(1 \nabla b)^{\prime}\right]^{\prime}$ where $b \neq 0, a \cdot 0=0$. Clearly, $a \cdot b$ is in $K$ for every $a$ and $b$ in $K$.

$\mathrm{T}_{17}^{\prime} \cdot a \cdot b=\{1 \nabla\{[((1 \nabla b) \nabla 1) \nabla a] \nabla 1\}\} \nabla 1$ (where $\left.a, b \neq 0\right)$.

Proof. All combinations indicated in the theorem are in $K$ by $\mathrm{T}_{12}^{\prime}, \mathrm{T}_{7}^{\prime}$, 
$\mathrm{F}_{6}^{\prime}, \mathrm{T}_{4}^{\prime}$, and so on.

$$
\begin{aligned}
a \cdot b & =\left[a \nabla(1 \nabla b)^{\prime}\right]^{\prime}=\left\{(a \nabla a)^{\prime} \nabla\left[(1 \nabla b)^{\prime} \nabla a\right]^{\prime}\right\}^{\prime}=\left\{1 \nabla\left[(1 \nabla b)^{\prime} \nabla a\right]^{\prime}\right\}^{\prime} \\
& =\{1 \nabla\{[([1 \nabla b] \nabla 1) \nabla a] \nabla 1\}\} \nabla 1 \quad b y \quad \mathrm{D}_{6}^{\prime}, \mathrm{T}_{14}^{\prime}, \mathrm{D}_{4}^{\prime}, \mathrm{T}_{9}^{\prime} .
\end{aligned}
$$

$\mathrm{D}_{7}^{\prime} \cdot a-b=a \cdot(b \nabla a)$, where $a \neq 0$. (The definition of $0-b$ is not supplied here because it involves considerable complexity unnecessary for the establishment of sufficiency. Clearly, $a-b$ is in $K$ for every $a$ and $b$ in $K$ except $a=0$.

$$
\left.\mathrm{T}_{18}^{\prime} . a-b=0 \text { if and only if } a=b \text { (where } a \neq 0\right) \text {. }
$$

Proof. $a-a=a \cdot(a \nabla a)=a \cdot 0=0$ by $\mathrm{D}_{7}^{\prime}, \mathrm{D}_{2}^{\prime}, \mathrm{D}_{6}^{\prime}$. Next, let $a-b=0$. Then $a \cdot(b \nabla a)=0$. If $b \nabla a \neq 0,\left\{a \nabla[1 \nabla(b \nabla a)]^{\prime}\right\}^{\prime}=0$ by $\mathrm{D}_{6}^{\prime}$, which is impossible since $a \neq 0$. Therefore $b \nabla a=0$ and $a=b$.

$$
\left.\mathrm{T}_{19}^{\prime} . a-b=\left[(b \nabla a) \nabla(1 \nabla a)^{\prime}\right]^{\prime} \text { (where } a \neq 0\right) \text {. }
$$

Proof. Let $a \neq 0$. For $a=b$ the theorem follows from $\mathrm{T}_{18}^{\prime}$. Let $a \neq b$. Then $b \nabla a \neq 0$ and $[1 \nabla(b \nabla a)]^{\prime} \nabla a=(1 \nabla a)^{\prime} \nabla(b \nabla a)$ by $\mathrm{T}_{15}^{\prime}$. Hence, $a \nabla[1 \nabla(b \nabla a)]^{\prime}$ $=(b \nabla a) \nabla(1 \nabla a)^{\prime}(1)$; by $\mathrm{T}_{8}^{\prime} \cdot a-b=a \cdot(b \nabla a)=\left\{a \nabla[1 \nabla(b \nabla a)]^{\prime}\right\}^{\prime}=[(\mathrm{b} \nabla \mathrm{a})$ $\left.\nabla(1 \nabla a)^{\prime}\right]^{\prime}$ by $\mathrm{D}_{7}^{\prime}, \mathrm{D}_{6}^{\prime},(1)$.

\section{$\mathrm{T}_{20}^{\prime} .\left[(a \nabla b) \nabla(1 \nabla b)^{\prime}\right]^{\prime} \nabla c=(a \nabla b) \nabla(c \nabla b)^{\prime}($ where $b, c \neq 0)$.}

Proof. For $a=b$ the theorem is obvious by $\mathrm{T}_{b}^{\prime}$. Let $a \neq b$ and $b, c \neq 0$. By $\mathrm{T}_{12}^{\prime}$ and $\mathrm{T}_{4}^{\prime}$ all the following combinations of elements are in $K$. $\left[(a \nabla b) \nabla(1 \nabla b)^{\prime}\right]^{\prime} \nabla(a \nabla b)=[(a \nabla b) \nabla(a \nabla b)]^{\prime} \nabla(1 \nabla b)^{\prime}=1 \nabla(1 \nabla b)^{\prime}=b^{\prime}=c \nabla(c \nabla b)^{\prime}$ by $\mathrm{T}_{16}^{\prime}, \mathrm{D}_{4}^{\prime}, \mathrm{T}_{13}^{\prime}, \mathrm{T}_{13}^{\prime}$. Hence, $\left[(a \nabla b) \nabla(1 \nabla b)^{\prime}\right] \nabla c=(a \nabla b) \nabla(c \nabla b)^{\prime}$ by $\mathrm{F}_{3}^{\prime}$.

$$
\left.\mathrm{T}_{21}^{\prime} . a-(b-c)=c-(b-a) \text { (where } a, b, c \neq 0\right) \text {. }
$$

Proof. Let $a, b, c \neq 0$ and suppose first that $c \nabla b=(a \nabla b)^{\prime}$ (1). Then $b-c$ $=\left[(c \nabla b) \nabla(1 \nabla b)^{\prime}\right]^{\prime}=\left[(a \nabla b)^{\prime} \nabla(1 \nabla b)^{\prime}\right]^{\prime}=(a \nabla 1)^{\prime}=a^{\prime \prime}=a$ by $\mathrm{T}_{19}^{\prime},(1), \mathrm{T}_{14}^{\prime}, \mathrm{T}_{9}^{\prime}$, $\mathrm{T}_{11}^{\prime}$. Similarly, $b-a=c$ and the theorem is obvious from $\mathrm{T}_{18}^{\prime}$.

Next let $c \nabla b \neq(a \nabla b)^{\prime}$. Then $(c \nabla b) \nabla(a \nabla b)^{\prime} \neq 0$. Hence $\left[(c \nabla b) \nabla(1 \nabla b)^{\prime}\right]^{\prime} \nabla a \neq 0$ by $T_{20}^{\prime}$. Also $(1 \nabla a)^{\prime},(a \nabla b)^{\prime},(c \nabla b)^{\prime} \neq 0$ by $T_{12}^{\prime}$. Hence all the following expressions are in $K$.

$$
\begin{aligned}
\left\{\left[(a \nabla b) \nabla(1 \nabla b)^{\prime}\right]^{\prime} \nabla c\right\} \nabla(a \nabla b)^{\prime} & =\left[(a \nabla b) \nabla(c \nabla b)^{\prime}\right] \nabla(a \nabla b)^{\prime}=\left[(a \nabla b)^{\prime \prime} \nabla(c \nabla b)^{\prime}\right] \nabla(a \nabla b)^{\prime} \\
& =\left[(c \nabla b)^{\prime \prime} \nabla(a \nabla b)^{\prime}\right] \nabla(c \nabla b)^{\prime}=\left[(c \nabla b) \nabla(a \nabla b)^{\prime}\right] \nabla(c \nabla b)^{\prime} \\
& =\left\{\left[(c \nabla b) \nabla(1 \nabla b)^{\prime}\right]^{\prime} \nabla a\right\} \nabla(c \nabla b)^{\prime}
\end{aligned}
$$

by $T_{20}^{\prime}, T_{11}^{\prime}, F_{6}^{\prime}, T_{11}^{\prime}, T_{20}^{\prime}$. From the first and last members,

$$
\left\{\left[(a \nabla b) \nabla(1 \nabla b)^{\prime}\right]^{\prime} \nabla c\right\} \nabla\left\{\left[(c \nabla b) \nabla(1 \nabla b)^{\prime}\right]^{\prime} \nabla a\right\}=(a \nabla b)^{\prime} \nabla(c \nabla b)^{\prime}=a \nabla c
$$


(2) by $\mathrm{F}_{3}^{\prime}, \mathrm{T}_{14}^{\prime}$. But $(1 \nabla c)^{\prime} \nabla a=(1 \nabla a)^{\prime} \nabla c$ by $\mathrm{T}_{15^{\prime}}$. Hence $a \nabla c=(1 \nabla c)^{\prime} \nabla(1 \nabla a)^{\prime}$

(3) by $F_{3}^{\prime}$ so that

$$
\left\{\left[(a \nabla b) \nabla(1 \nabla b)^{\prime}\right]^{\prime} \nabla c\right\} \nabla(1 \nabla c)^{\prime}=\left\{\left[(c \nabla b) \nabla(1 \nabla b)^{\prime}\right]^{\prime} \nabla a\right\} \nabla(1 \nabla a)^{\prime}
$$

(4) by (2), (3), $F_{3}^{\prime}$. Therefore

$$
\begin{aligned}
c-(b-a) & =\left\{\left\{\left[(a \nabla b) \nabla(1 \nabla b)^{\prime}\right]^{\prime} \nabla c\right\} \nabla(1 \nabla c)^{\prime}\right\}^{\prime} \\
& =\left\{\left\{\left[(c \nabla b) \nabla(1 \nabla b)^{\prime}\right]^{\prime} \nabla a\right\} \nabla(1 \nabla a)^{\prime}\right\}^{\prime}=a-(b-c)
\end{aligned}
$$

by $\mathrm{T}_{19^{\prime}},(4), \mathrm{T}_{19^{\prime}}$.

$\mathrm{T}_{22}^{\prime} .(a-b)-(c-d)=(a-c)-(b-d)$ (where $a, b, c \neq 0$ and $\left.a \neq b, c\right)$.

Proof. First let $d \neq 0$. From $a \neq b, c$ follows $a-b \neq 0, a-c \neq 0$; by $\mathrm{T}_{18}$. Hence the following expressions are all in $K$ by $\mathrm{D}_{7}^{\prime}$. Then,

$(a-b)-(c-d)=d-[c-(a-b)]=d-[b-(a-c)]=(a-c)-(b-d) ;$

by $\mathrm{T}_{21}^{\prime}, \mathrm{T}_{21}^{\prime}, \mathrm{T}_{21}^{\prime}$. Next let $d=0$. Note that for any element $e, e-0=e(1)$; for $e-0=e \cdot(0 \nabla e)=e \cdot 1=\left[e \nabla(1 \nabla 1)^{\prime}\right]^{\prime}=e^{\prime \prime}=e$ by $\mathrm{D}_{7}^{\prime}, \mathrm{D}_{6}^{\prime}, \mathrm{T}_{11}^{\prime}$. Hence $b-d=b$, $c-d=c$ (2). If $a-b=c$ (3) then $b-(a-c)=c-(a-b)=c-c=0$ by $\mathrm{T}_{21}^{\prime}$, (3), $T_{18}^{\prime}$. Hence, $a-c=b(4)$ by $\mathrm{T}_{18}^{\prime}$. Then $(a-b)-c=c-c=0=(b-b)=(a-c)-b$ by (3), $\mathrm{T}_{18}^{\prime}, \mathrm{T}_{18}^{\prime},(4)$. Finally, let $a-b \neq c$. Then, as above, $a-c \neq b$ and all the following expressions are in $K$. Therefore $[(a-b)-c]-[(a-c)-b]=b-\{(a-c)$ $-[(a-b)-c]\}=b-\{c-[(a-b)-(a-c)]\}=b-\{c-[c-(a-(a-b))]\}$ $=b-\{c-[c-(b-(a-a))]\}=b-\{c-[c-(b-0)]\}=b-[c-(c-b)]$ $=b-[b-(c-c)]=b-(b-0)=b-b=0$; by $\mathrm{T}_{21}^{\prime}, \mathrm{T}_{21}^{\prime}, \mathrm{T}_{21}^{\prime}, \mathrm{T}_{21}^{\prime}, \mathrm{T}_{18}^{\prime},(1), \mathrm{T}_{21}^{\prime}$, $\mathrm{T}_{18}^{\prime},(1), \mathrm{T}_{18}^{\prime}$. Hence $(a-b)-c=(a-c)-b$ and the theorem holds by $\mathrm{T}_{18}^{\prime},(2)$.

6. Sufficiency of $(F)$ and $\left(F^{\prime}\right)$ for fields. It will be convenient to establish the sufficiency of $\left(F^{\prime}\right)$ first by deriving Wiener's postulates from $\left(F^{\prime}\right)$. The sufficiency of $(F)$ is then readily proved by deriving $\left(F^{\prime}\right)$ from $(F)$. Following are Wiener's postulates and their derivation from $\left(\mathrm{F}^{\prime}\right)$.

I. "Whatever $x$ may be, there is a $K$-element $y$ such that $x \nabla(y \nabla y)$ is not a $K$-element."

Proof. Take $y \neq 0$. Then $y \nabla y=0$ by $\mathrm{D}_{2}^{\prime}$. Hence $x \nabla(y \nabla y)$ is not in $K$ by $\mathrm{T}_{4}^{\prime}$.

II. "If $x$ and $y$ are $K$-elements, but $x \nabla y$ is not a $K$-element, there is a $K$-element $z$ such that $y=z \nabla z$."

Proof. If $x \nabla y$ is not a $K$-element, $y=0$ by T $T_{4}^{\prime}$. Take $z \neq 0$. Then $y=z \nabla z$ by $\mathrm{D}_{2}^{\prime}, \mathrm{T}_{4}^{\prime}$.

III. "Whenever $x$ and $y$ are distinct $K$-elements, either $x \nabla y$ or $y \nabla x$ is a $K$-element."

Proof. Since $x \neq y$, either $x \neq 0$ or $y \neq 0$ by $\mathrm{T}_{3}^{\prime}, \mathrm{D}_{2}^{\prime}$. Then $y \nabla x$ or $x \nabla y$ is an element of $K$ accordingly as $x \neq 0$ or $y \neq 0$ by $\mathrm{T}_{4}^{\prime}$. 
IV. "Whenever $x, y, u, v$, and their indicated combinations are $K$-elements, and $x \nabla y=u \nabla v$, then $x \nabla u=y \nabla v$."

Proof. The proof is by $\mathrm{F}_{3}^{\prime}$.

Definition A. " $Z=x \nabla x$ if $x$ and $x \nabla x$ belong to $K$, and $x \nabla x$ is unique in the system."

Note that $Z=0$ by $\mathrm{T}_{3}^{\prime}, \mathrm{D}_{2}^{\prime}$.

V. "If $x, y, x \nabla y$, and $Z$ are $K$-elements, and $x \nabla y=Z$, then $x=y$."

Proof. The proof is by $\mathrm{T}_{6}^{\prime}$.

VI. "If $x, y, z, Z$, and their indicated combinations belong to $K$,

$$
[(x \nabla y) \nabla(Z \nabla y)] \nabla z=[(x \nabla z) \nabla(Z \nabla z)] \nabla y . "
$$

Proof. The proof is by $T_{16}^{\prime}$.

Definition B. " $U=Z \nabla x$, if $x, Z$, and $Z \nabla x$ belong to $K$, and $Z \nabla x$ is unique in the system."

Note that $U=1$ by $\mathrm{T}_{6}^{\prime}, \mathrm{D}_{3}^{\prime}$.

Definition C. " $x \odot y=\{1 \nabla\{[((1 \nabla y) \nabla 1) \nabla x] \nabla 1\}\} \nabla 1$, if all the indicated expressions belong to $K$. Otherwise, if $Z$ belongs to $K, x \odot y=Z$."

Note that $x \odot y=x \cdot y$; for, if the indicated expressions in $x \odot y$ are all in $K$, $x, y \neq 0$ by $\mathrm{T}_{4}^{\prime}$. Hence in this case $x \odot y=x \cdot y$ by $\mathrm{T}_{17}$. If not all the indicated expressions for $x \odot y$ are in $K$, either $x=0$ or $y=0$ by $\mathrm{T}_{4}^{\prime}$. For $y=0, x \odot 0=0$ $=x \cdot 0$ by $\mathrm{D}_{6}^{\prime}$. For $x=0,0 \odot y=0=\left[0 \nabla(1 \nabla y)^{\prime}\right]^{\prime}=0 \cdot y$ by $\mathrm{T}_{10} \mathrm{D}^{\prime}, \mathrm{D}_{6}^{\prime}$.

DEFINITION D. " $x \sim y=x \odot(y \nabla x)$."

Note that $x \sim y=x-y$ by $D_{7}^{\prime}$, if $x \neq 0$. Neither $0 \sim y$ nor $0-y$ has been defined.

VII. "If $x, y, u, v$, and their indicated combinations belong to $K$,

$$
(x \sim y) \sim(u \sim v)=(x \sim u) \sim(y \sim v) . ”
$$

Proof. The proof is by $\mathrm{T}_{22}^{\prime}$.

This establishes the sufficiency of $\left(\mathrm{F}^{\prime}\right)$ for fields.

To establish the sufficiency of $(F)$ by deriving $\left(F^{\prime}\right)$ from $(F)$ we have only to notice that $F_{1}^{\prime}$ to $F_{6}^{\prime}$ correspond respectively to $T_{13}$ to $T_{18}$ inclusive. $F_{3}^{\prime}$ to $\mathrm{F}_{6}^{\prime}$ are subject to the condition that the expressions involved are elements of $K$, but by $T_{11}$ this is precisely the significance of the conditions in $T_{15}$ to $T_{18}$. Hence $(F)$ is also sufficient for fields.

7. Necessity of the postulates of $(F)$ and $\left(F^{\prime}\right)$. The postulates of $(F)$ and $\left(\mathrm{F}^{\prime}\right)$ are all necessary for fields and can be derived from any of the well known postulate sets $\left(^{3}\right)$ in terms of $(+)$ and $(\cdot)$ by means of the definitions $a \Delta b=a \cdot(1-b)$ and $a \nabla b=1-a / b$.

8. Independence of the postulates. The independence of the postulates of $(F)$ and $\left(F^{\prime}\right)$ are established by the systems in the following table, all of which

(3) See, for example, E. V. Huntington, Note on the definitions of abstract groups and fields by sets of independent postulates, Trans. Amer. Math. Soc. vol. 6 (1905) pp. 181-193. 
are finite, it will be noted. $S_{i}$ is the independence system for $F_{i}$. The blanks indicate that the result of the corresponding combination is not in $K$.

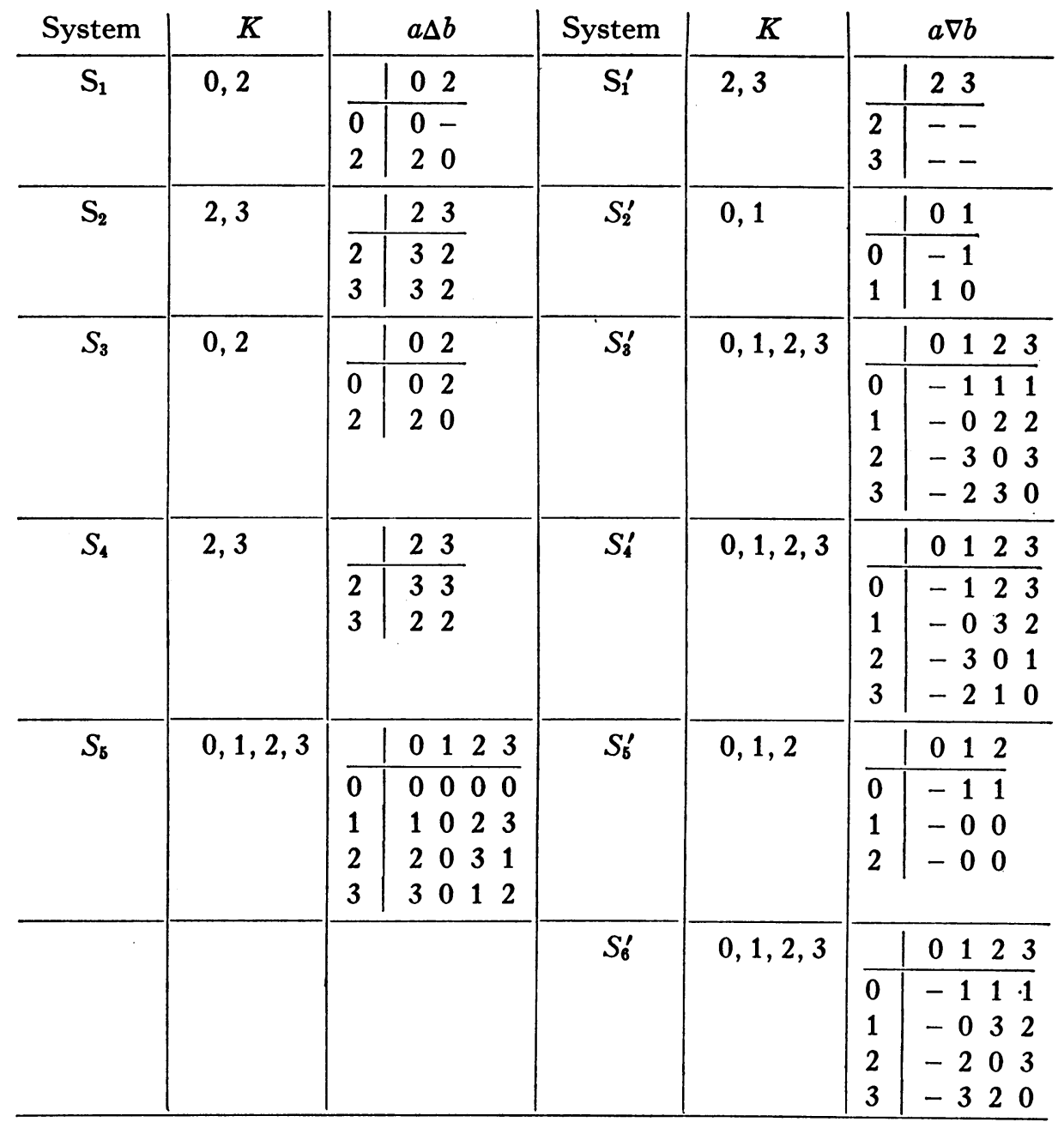

To these may be added an independence system for $N$, the same for both $(F)$ and $\left(F^{\prime}\right) . K$ consists of the single element 0 with $0 \Delta 0=0$ and $0 \nabla 0=0$.

9. Additional properties of the operations $\Delta$ and $\nabla$. The operations $\Delta$ and $\nabla$ have other interesting properties which were not brought.out in $\S \S 3,5$, since they were not required for the proofs of sufficiency. Some of these properties are stated below without proof:

(1) $0^{\prime}=1,1^{\prime}=0$. 
(2) $a^{\prime \prime}=a$. in fact,

(1) and (2) are true when $a^{\prime}$ is defined as in $\S 3\left(D_{4}\right)$ as well as $\S 4\left(D_{1}^{\prime}, T_{9}^{\prime}\right)$
(3) $1 \Delta a=a \nabla 1=a^{\prime}$.
(4) $a^{\prime} \Delta b=b^{\prime} \Delta a, a \Delta b^{\prime}=b \Delta a^{\prime}, a^{\prime} \Delta b^{\prime}=b \Delta a$.
(5) If $a \Delta b=a$ and $a \neq 0$, then $b=0$.
(6) If $a \Delta b=0$ and $a \neq 0$, then $b=1$.

Definition. $\bar{a}$ is the element uniquely determined by the equation $a \Delta \bar{a}=1$, when $a \neq 0$.

(7) The equation $a \Delta x=b$ has the unique solution $x=(b \Delta \bar{a})^{\prime}$ if $a \neq 0$.

(8) The equation $y \Delta a=b$ has the unique solution $y=b \Delta \overline{a^{\prime}}$ if $a \neq 1$.

The ordinary field operations are definable in terms of $\Delta$ as follows:

(A) $a \cdot b=a \Delta b^{\prime}$.

(B) $a / b=a \Delta b, b \neq 0$.

(C) $a-b=a \Delta p^{\prime}$ for $a \neq 0$, where $p$ is uniquely determined by the equation $a \Delta p=b .0-b=(c-b)-c$, where $c \neq 0, b$ and provided $K$ contains more than two elements. When $K$ consists of two elements, $0-b=b$.

(D) $a+b=(p \Delta a)^{\prime}$ for $a \neq 1$, where $p$ is uniquely determined by the equation $a^{\prime} \Delta p=b .1+b=1-(0-b)$.

As alternative definitions of subtraction and division we have:

(E) $a-b=a \Delta(b \Delta \bar{a})$ (where $a \neq 0)$.

(F) $a+b=\left[\left(b \Delta \overline{a^{\prime}}\right)^{\prime} \Delta a\right]^{\prime}$ (where $\left.a \neq 1\right)$.

(9) $a^{\prime}=1-a$.

(10) $a \Delta b=a-a \cdot b=a \cdot(1-b)$.

All rational operations, as well as the special elements 0 and 1 , can be expressed directly in terms of $\nabla$ without postulating any inverse operations. How this can be done for 0,1 , multiplication, and subtraction has already been shown. (See $\S 5, \mathrm{D}_{2}^{\prime}, \mathrm{D}_{3}^{\prime}, \mathrm{D}_{6}^{\prime}$, and $\mathrm{D}_{7}^{\prime}$, or $\mathrm{T}_{19^{\prime}}$.) Addition and division can be defined as follows:

(G) $a / b=(a \nabla b)^{\prime}, b \neq 0$.

(H) $a+b=\left(b \nabla a^{\prime}\right) \nabla\left(1 \nabla a^{\prime}\right)^{\prime}, a \neq 1$.

With / defined as in $(\mathrm{G})$ we have:

(11) $a \nabla b=(b-a) / b=1-a / b$.

(12) The unique solution of the equation $a \nabla x=b$, where $a, b \neq 0$, is $x=\left(a \nabla b^{\prime}\right)^{\prime}$.

(13) The unique solution of the equation $y \nabla a=b$, where $a, b \neq 0$, is $y=\left[a \nabla\left(1 \nabla b^{\prime}\right)^{\prime}\right]^{\prime}$.

The relationships between $\nabla$ and $\Delta$ are given by the following:

(14) $a \nabla b=(a \Delta b)^{\prime}$.

(15) $a \Delta b=\left[a \nabla\left(1 \nabla b^{\prime}\right)^{\prime}\right]^{\prime}$.

10. Equivalent field operations. In $\S 1$ it was stated that the simplest expression for $a \nabla b$ in terms of the usual field operations is $1-a / b$. By this was meant that $a \nabla b$ possesses the same formal properties as $1-a / b$. Moreover, 
with / and - defined in terms of $\nabla$, we find that $a \nabla b=1-a / b$ (see $\$ 9:$ (11)). However, the properties $\mathrm{F}_{1}^{\prime}-\mathrm{F}_{6}^{\prime}$ do not by any means characterize $\nabla$ uniquely. For example, $\mathrm{F}_{1}^{\prime}-\mathrm{F}_{\mathrm{b}}^{\prime}$ are all satisfied when $a \nabla b$ is interpreted as $(1-a) /(1-b)$. Furthermore, it is possible to give definitions different from the previous ones for - and / preserving the field properties of subtraction and division, yet in terms of which $a \nabla b=(1-a) /(1-b)$. These definitions are:

$$
a / b=a^{\prime} \nabla b^{\prime}, \quad b \neq 0 . \quad a-b=\left(b^{\prime} \nabla a^{\prime}\right) \nabla\left(1 \nabla a^{\prime}\right)^{\prime}, \quad a \neq 1 .
$$

All these facts can easily be verified. Similar considerations show that $a \Delta b$ can be interpreted as $a \cdot b-b+1$ just as well as $a \cdot(1-b)$. In short, $1-a / b$ and $(1-a) /(1-b)$ are in a certain sense formally equivalent to each other as are $a \cdot(1-b)$ and $a \cdot b-b+1$. We now proceed to give a general definition of this concept of equivalence and apply it to field operations.

Let $S$ be a mathematical system having a class $K$ and an ordered set of $m n$-ary operations $\left(O^{\prime}, O^{\prime \prime}, \cdots, O^{(m)}\right)$ as its primitives, and satisfying certain postulates $P_{1}-P_{k}$. If two ordered sets of $m n$-ary operations, $\left(\Delta^{\prime}, \Delta^{\prime \prime}, \cdots, \Delta^{(m)}\right)$ and $\left(\nabla^{\prime}, \nabla^{\prime \prime}, \cdots, \nabla^{(m)}\right)$, both satisfy the postulates $P_{1}-P_{k}$, these sets of operations are said to be equivalent:

$$
\left(\Delta^{\prime}, \Delta^{\prime \prime}, \cdots, \Delta^{(m)}\right) \sim\left(\nabla^{\prime}, \nabla^{\prime \prime}, \cdots, \nabla^{(m)}\right) .
$$

This notion of equivalence is obviously reflexive, symmetric, and transitive. It is also clear that all the theorems deducible from $P_{1}-P_{k}$ will remain true if the operations of one set are replaced throughout by those of an equivalent set.

As an example, consider the pairs of operations, $\left(\oplus^{\prime}, \odot^{\prime}\right)$ and $\left(\oplus^{\prime \prime}, \odot^{\prime \prime}\right)$, given by

$$
\begin{array}{ll}
a \oplus^{\prime} b=a+b+1, & a \odot^{\prime} b=a+b+a \cdot b, \\
a \oplus^{\prime \prime} b=a+b-1, & a \odot^{\prime \prime} b=a+b-a \cdot b .
\end{array}
$$

Since, as is easily seen, both pairs satisfy the Huntington postulates for fields in terms of + and $\cdot($ footnote 3 ), we have

$$
\left(\oplus^{\prime}, \odot^{\prime}\right) \sim\left(\oplus^{\prime \prime}, \odot^{\prime \prime}\right) \sim(+, \cdot) .
$$

The totality of such operations is given by the following theorem.

THEOREM I. The most general pair of rational binary operations $(\oplus, \odot)$ equivalent to the sum and product $(+, \cdot)$ in every field are of the form

$$
\begin{aligned}
& a \oplus b=a+b-Z, \\
& a \odot b=\frac{a b-Z(a+b)+U Z}{U-Z},
\end{aligned}
$$

where the parameters $U$ and $Z$ are distinct arbitrary field elements. 
Proof. It can be readily verified that for each $U$ and $Z$ the operations (1) and (2) are equivalent to $a+b$ and $a \cdot b$ by observing that they satisfy the Huntington field postulates. To show that they are the most general such operations, let $a \oplus b$ and $a \odot b$ be any pair of rational functions of $a$ and $b$ equivalent to $a+b$ and $a \cdot b$ in an arbitrary field $F$. Then any field property of + and - must also be true of $\oplus$ and $\odot$. To eliminate an operation from consideration we need only exhibit a particular field in which it does not possess one of these properties. Thus, $a \oplus b$ and $a \odot b$ must be elements of $F$ for every $a$ and $b$ in $F$. But in the field of complex numbers values of $a$ and $b$ can always be found to make the denominator of any rational non-integral function of $a$ and $b$ vanish, and for this $a$ and $b$ the value of the function fails to be in the field. Hence, we may restrict our attention to operations expressible as polynomials in $a$ and $b$.

Next, the equations

$$
\begin{array}{ll}
\alpha \oplus x=\beta, & x \oplus \alpha=\beta, \\
\gamma \odot x=\delta, & x \odot \gamma=\delta
\end{array}
$$

must have unique solutions in $x$ for all $\alpha, \beta, \delta$ and for all $\gamma$ except the identity element of $\oplus$. This enables us to say that $a \oplus b$ and $a \odot b$ must be of the form

$$
\begin{aligned}
& a \oplus b=A a b+B a+C b+D, \\
& a \odot b=M a b+P a+Q b+R,
\end{aligned}
$$

where $A, B$, and so on, are arbitrary field elements subject to the conditions

$$
A=0 \text { implies } B, C \neq 0, \text { and } M=0 \text { implies } P, Q \neq 0 .
$$

For, if $a \oplus b$ contained a term in $b^{n}, n>1$, for example, then the equations (3) would have $n>1$ solutions in the field of complex numbers; while, if $M$ and $P$ were both zero, for instance, $a \odot b$ would contain only $b$, and the second of the equations (4) would be solvable only when $\delta=Q \gamma+R$. We can further assert that $A=0$ in (5). For, if $A \neq 0$, the first equation (3) would have no solution when $\alpha=-C / A$ and $\beta=B \alpha+D$, since under these circumstances $\alpha \oplus x$ reduces to $B \alpha+D$. This argument cannot be used to prove $M=0$ because of the exceptional value of $\gamma$ for which (4) need not have a solution.

To obtain further conditions on the coefficients of (5) we use the commutative, associative, and distributive laws of addition and multiplication. From the first of these we find, for example,

$$
A a b+B a+C b+D=A b a+B b+C a+D
$$

identically in $a$ and $b$. From this and the corresponding identity for $a \odot b$, we get, by equating coefficients of like terms,

$$
B=C, \quad P=Q \text {. }
$$


Using (8) and making similar application of the associative and distributive laws respectively, we obtain

$$
\begin{aligned}
B^{2} & =B, \\
P^{2} & =P+M R, \\
P & =M D, \\
D P & =R+D .
\end{aligned}
$$

Since $A=0, B \neq 0$ by (6). Hence, (9) implies $B=1$. (This fact was used in deriving (11) and (12).) The last three equations are not independent; elimination of $D$ between (11) and (12) yields (10). Note that $M \neq 0$; for, if it were, we should also have $P=0$ by (11) contrary to (6). We define $Z$ and $U$ as follows:

$$
Z=-D, \quad U=1 / M-D .
$$

From (11), (12), and (13) we find

$$
D=-Z, \quad M=1 /(U-Z), \quad P=-Z /(U-Z), \quad R=U Z /(U-Z),
$$

where obviously $U \neq Z$. Substituting from (14) and (8) into (5), we obtain (1) and (2).

COROLlaRY 1. The identity elements of the operations (1) and (2) are $Z$ and $U$ respectively.

COROLlARY 2. The most general pair of rational operations equivalent to subtraction and division in every field (that is, the inverses of the operations (1) and (2)) are

$$
\begin{aligned}
& a \ominus b=a-b+Z, \\
& a \varnothing b=\frac{(U-Z) \cdot a+Z b-U Z}{b-Z},
\end{aligned}
$$

TheOREM II. The most general rational operations satisfying the postulates of $(\mathrm{F})$ and $\left(\mathrm{F}^{\prime}\right)$ respectively are

$$
\begin{aligned}
& a \Delta^{\prime} b=a \ominus a \odot b=\frac{U a+Z b-a b-Z^{2}}{U-Z}, \\
& a \nabla^{\prime} b=(b \odot a) \varnothing b=\frac{U b-(U-Z) a-Z^{2}}{b-Z}
\end{aligned}
$$

where $U$ and $Z$ are distinct arbitrary field elements.

The proof is left to the reader.

11. Other single primitive field operations. As examples of other single operations that can be taken as primitive in a definition of fields, there may 
be mentioned the following:

$$
a \diamond b=a /(a-b) \text { and } a \square b=(a b+a-b) /(a b-b) .
$$

Postulate sets for fields in terms of these operations will not be stated in detail here. However, by the following methods $\nabla$ may be expressed in terms of $\diamond$ and $\square$. Once $\nabla$ has been defined, the usual field operations $(+,-, \cdot$, and /) can be expressed in terms of $\nabla$ as in $\$ \S 5$ and 9.

Let $a$ and $b$ be any $K$-elements. 1 and 0 are defined as the unique $K$-elements such that $a \square 0$ and $1 \square b$ are not in $K . b$ designates the unique element determined for each $b$ by $(b \square b) \square b=0, b \neq 0$. Then, $a \nabla b=(a \square a) \square b, a \neq 1,0$; $b \neq 0$.

$a \diamond b$ is in $K$ if and only if $a \neq b .0$ is defined as the unique element such that $0 \diamond b=0$ for all $b .1=a \diamond 0, a \neq 0$. Then, $a \nabla b=1 \diamond(a \diamond b), b \neq 0, a$.

Finally there remains the problem of determining the necessary and sufficient conditions an operation $O$ must satisfy in order that a definition of field may be formulated with $O$ as the only primitive operation. An equivalent statement of this problem is to determine all operations capable of generating the usual field operations in the general field. The solution depends on precisely what processes are to be admitted in generating derivative operations from the primitive operation. When $\nabla$ is taken as primitive, iteration is the only process required. When $\Delta$ is taken as primitive, iteration alone is not sufficient $\left({ }^{4}\right)$; inversion must also be permitted. A complete discussion of this problem would lead too far afield from our main objective, the development of specific postulate sets, and so will be left to a projected future paper. However, it may be mentioned that, for the case when only iteration is admissible, a solution, valid for a wide class of fields, has been obtained by $\mathrm{N}$. Wiener $\left(^{(5)}\right.$. In the general field his conditions on the primitive operation are necessary; but they are not sufficient, as the following example shows: $(a-b) /(a+b)$ satisfies Wiener's conditions and will in fact generate all rational functions over the field of rational numbers, for instance, but cannot generate the usual field operations over a field of characteristic 2, where it degenerates into the constant element 1.

Washington, D. C.

(4) A proof of this fact can be found in the reference of footnote 5 below.

(5) Norbert Wiener, Bilinear operations generating all operations rational in a domain $\Omega$, Ann. of Math. vol. 21 (1920) pp. 157-165. 